\title{
Transfer effects from single-task learning to simultaneous learning
}

\author{
BENTON J. UNDERWOOD \\ Northwestern University, Evanston, Illinois 60201
}

and

\author{
ARNOLD M. LUND \\ Bell Laboratories, Holmdel, New Jersey 07733
}

\begin{abstract}
Subjects learned three lists of words simultaneously. One list was recalled, one was tested by recognition, and the subjects made frequency judgments of the words in the third list. One group of subjects had one isolated learning-test trial on each of the three lists prior to two simultaneous learning trials. A second group had only the two simultaneous learning trials. Transfer from isolated to simultaneous learning was essentially perfect for the list that was recalled. However, the isolated learning trial resulted in very heavy negative transfer when recognition and when frequency estimations were used as response measures. In both cases, it was almost as if the subjects had not been given the isolated learning trial.
\end{abstract}

Simultaneous learning involves the acquisition of two or more lists of verbal units at once. For example, we might have a list of 30 animal names and a list of 30 vegetable names. To form a list for simultaneous free recall learning, the 30 words in each of the two lists would be paired randomly. Then, on a study trial, the subject is shown a pair of words, one from each category, then another pair, and so on, for the 30 presentations. On the test, the subjects recall each list independently.

In earlier work, we had used simultaneous learning as a means of studying differential encoding (Underwood \& Malmi, Note 1). The subjects were given three lists to learn simultaneously. They recalled one of the lists, made frequency judgments for another, and were tested by recognition on the third. The subjects were instructed ahead of time about the different materials and about the different tests. For reasons that are not germane here, in one of the experiments, we gave the subjects a study-test trial on each of the three lists alone before the lists were combined for simultaneous learning. It was found that there was strong positive transfer from isolated to simultaneous learning for the list that was recalled, but that there was substantial negative transfer for recognition and for frequency judgments. The present experiment is essentially a replication of the previous

This research was supported by the Personnel and Training Research Programs, Psychological Sciences Division, Office of Naval Research, under Contract N00014-78-C-0661, Contract Authority Identification NR 154-424. Reproduction in whole or in part is permitted for any purpose of the United States Government. Requests for reprints should be sent to Benton J. Underwood, Department of Psychology, Northwestern University, Evanston, Illinois 60201. study, but with completely new materials making up the lists.

\section{METHOD}

\section{Lists}

Three different lists were used, each associated with a different response measure. One list consisted of 21 pairs of men's and women's names (e.g., Allen-Marie). Each of these pairs was presented twice on a given study trial, and recall was required of the pair as a pair. A second list consisted of 42 two-word phrases (e.g., income-tax). The response measure for this list was recognition, consisting of a yes-no test in which the 42 "old" items occurred along with 24 "new" ones. The third list consisted of 21 animal names, and the memory test consisted of absolute frequency judgments for each of the animal names. In the study list, seven names were presented once, seven twice, and seven three times. These 21 words were mixed with seven new words on the tests.

\section{Procedure and Subjects}

A study trial consisted of 42 presentations. Each presentation consisted of one item from each of the three lists when simultaneous learning was involved. When lists were learned alone (in isolation), there were also 42 presentations, but the material shown was from only one list. For simultaneous learning, the presentation interval was $12 \mathrm{sec}$; for isolated learning, the interval was $4 \mathrm{sec}$. The order of the items on all study trials was the same.

There were two groups of 30 subjects each. One group received a single study-test trial on each of the three lists alone before the lists were combined for simultaneous learning. Two study-test trials of simultaneous learning followed the "alone" trials. This group will be identified as Group ATT, to indicate one trial alone and two trials for all lists together. The other group simply had two simultaneous learning trials and will be identified as Group TT.

The subjects were fully instructed from the beginning as to the various steps to be taken. The differences in the lists were described, and the subjects were told about the nature of the tests for each list. When the lists were learned alone by the 
subjects in Group ATT, the recall list was given first, the list of animals for frequency judgments second, and the recognition list third. The tests were also given in this order on the simultaneous learning trials.

\section{RESULTS}

The logic of the experiment calls for an examination of the comparative performance of the two groups on the simultaneous learning trials. In addition, however, we will show the performance on the A trial for each list.

It will be remembered that recall was requested for the pairs of names. We counted as correct only those pairs in which both members were given. The mean number correct on each trial is seen in Figure 1. The outstanding feature of the recall results is the dominance of study time in determining the performance and the lack of an effect of context (whether an A trial or a $T$ trial). For example, the performance of Group ATT on the first test trial of simultaneous learning is almost identical to the second test trial for Group TT. Thus, the subjects in Group ATT, having $4 \mathrm{sec}$ to study each pair on the A trial and $4 \mathrm{sec}$ to study each pair on the first $T$ trial (if study time was allotted equally to the three lists), recalled almost exactly the same number of pairs as did the subjects in Group TT on the second trial. It appears that transfer from isolated learning to simultaneous learning for this recall task was essentially $100 \%$.

For the frequency judgments, the number of hits was used as the response measure. This measure is the number

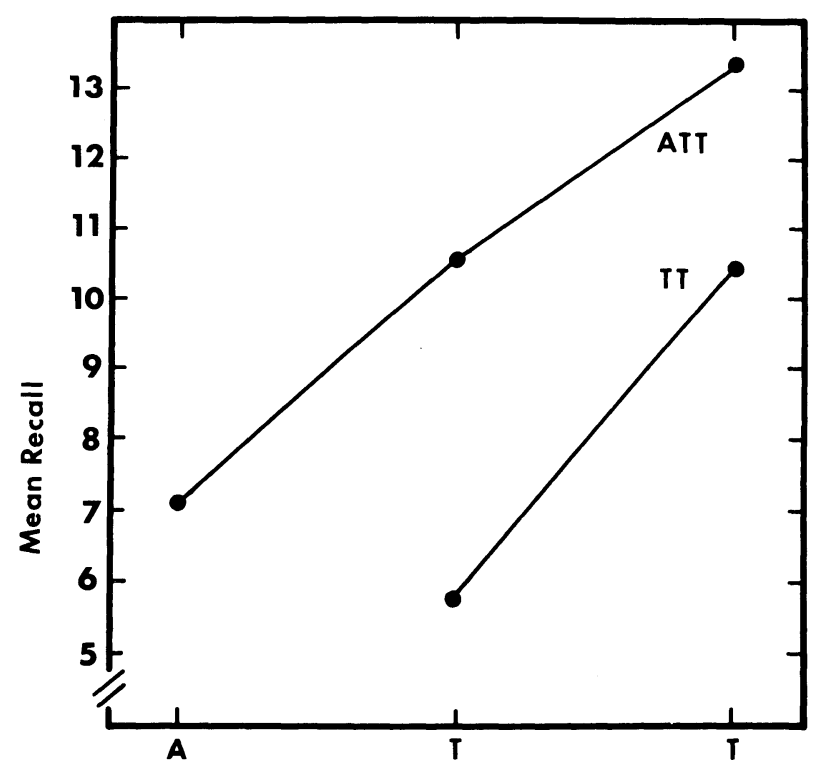

Trial

Figure 1. Recall in simultaneous learning with (ATT) and without (TT) an isolated study-test trial (A) before the two simultaneous learning trials.

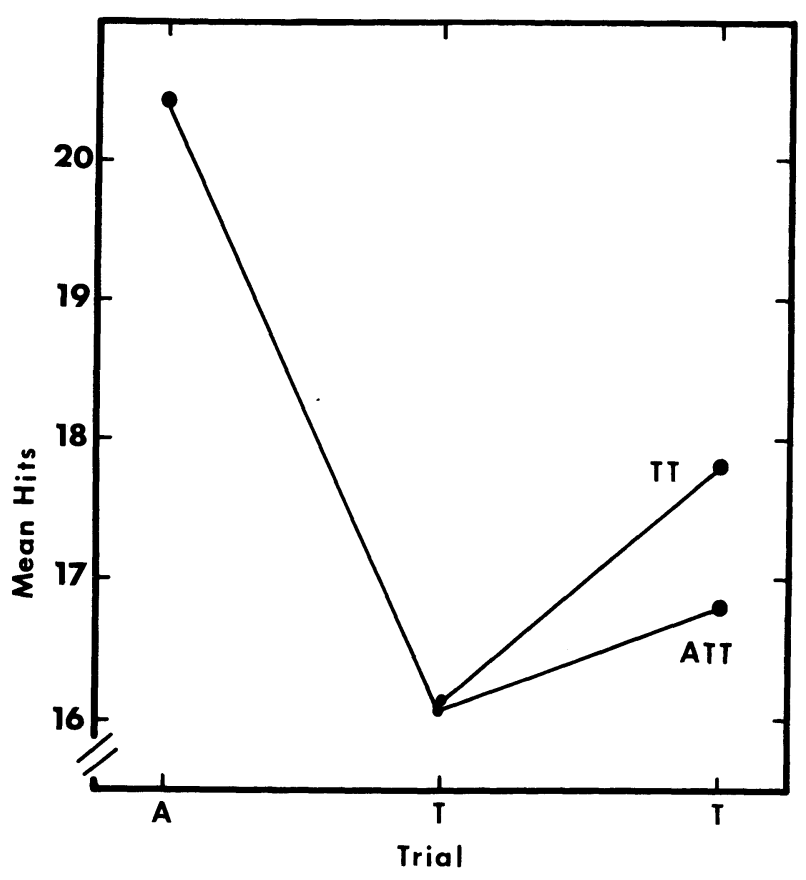

Figure 2. Mean hits of the frequency judgments with (ATT) and without (TT) a prior isolated study-test trial.

of cases (out of 28 possible) in which the subject identified the frequency correctly. The results are seen in Figure 2. The striking outcome here is the negative transfer that occurred in moving from the A trial to the first $\mathrm{T}$ trial for Group ATT. We know from previous work that the precision of frequency judgments does not increase much across trials (Underwood \& Malmi, 1978), but in this case we are dealing with very severe negative transfer from the A trial to the first $T$ trial. In fact, on the $T$ trials, performance was actually a little better for Group TT than for Group ATT. Overall, there was no evidence that the A trial for Group ATT had any enduring influence on the memory for frequency.

The recognition data are shown in Figure 3, with the false alarms plotted in the upper panel and the misses in the lower. Looking first at the misses, it can be seen that there was a negative influence resulting from having the A trial. For Group ATT, the mean misses actually increased from the A trial to the first $\mathrm{T}$ trial. However, the negative transfer was not complete. An analysis of variance of the two $T$ trials for the two groups showed Group TT to have lower performance (more misses) than Group ATT $[F(1,58)=4.46, \quad \mathrm{MSe}=38.77]$. In the case of the false alarms, the negative transfer was statistically complete, in that the two groups did not differ on the two $\mathrm{T}$ trials $(\mathrm{F}<1)$.

\section{DISCUSSION}

The results have indicated that recall learning was perfectly transferable from isolated to simultaneous learning, whereas 


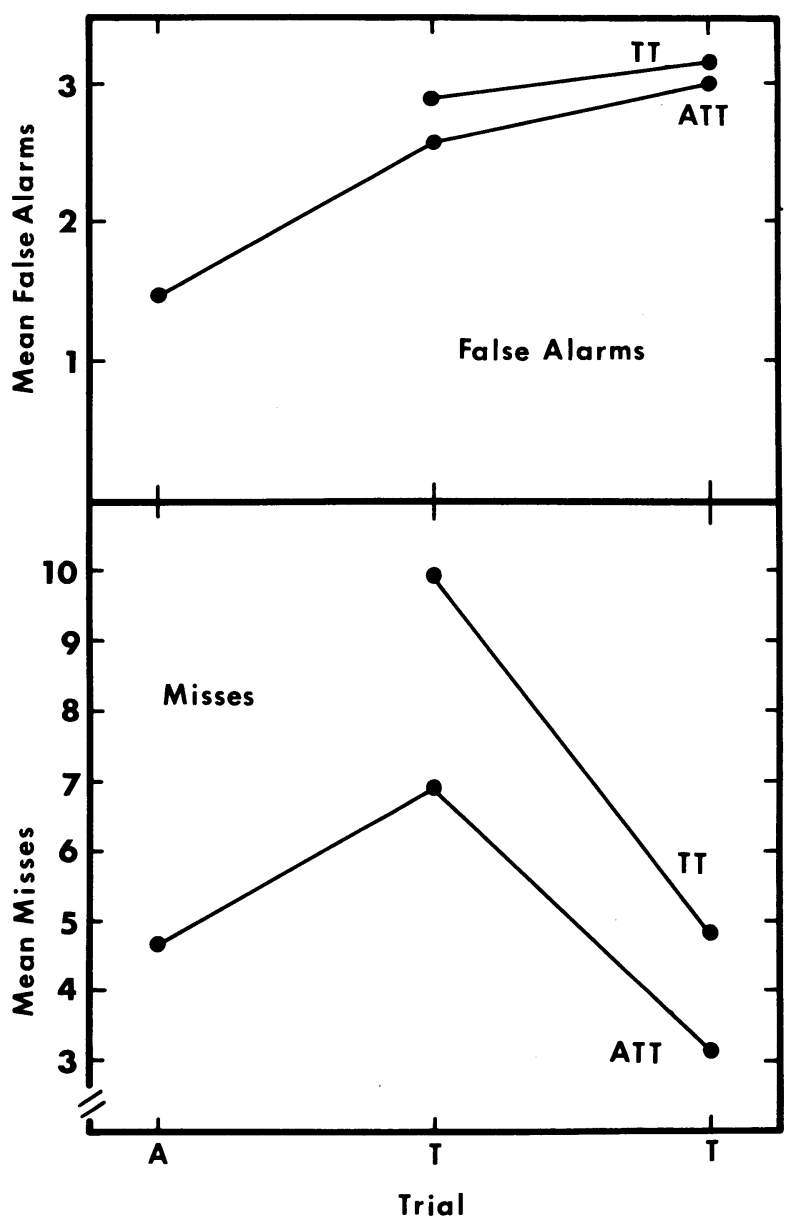

Figure 3. Mean misses and mean false alarms in simultaneous learning with (ATT) and without (TT) a prior isolated study-test trial. severe negative transfer was observed for recognition memory and frequency judgments. Thus, this is another case to be added to the many others in the literature that show that recall and recognition may well be based on different underlying mechanisms. The best single source for this evidence is Brown (1976).

We do not have an explanation for the findings. Moving from isolated learning to simultaneous learning could be viewed as a context change, but why this change should have such a powerful negative effect on recognition and frequency judgments and not on recall is not apparent to us. One possibility for the negative effects is that, as a consequence of the tests given on the lists following isolated learning, the so-called new items used on the tests for both recognition and frequency judgments become old when they appear on the second test, and hence they may be a source of false alarms. If this were true, however, it would seem that a comparable decrement would occur on the second test for the subjects in Group TT, because the new items used for the first test would become old for the second test. Nevertheless, we carried out a further experiment in which the critical group of subjects was given an A study trial on each list prior to simultaneous learning but no tests were given until after the first simultaneous learning trial. Under these circumstances, a new item did not become old for the test given after the first simultaneous learning trial. This did not change the results for recognition and for frequency judgments. In both cases, there was heavy negative transfer.

\section{REFERENCE NOTE}

1. Underwood, B. J., \& Malmi, R. A. Recall and recognition of tasks learned simultaneously (ONR Technical Report). Evanston, Ill: Northwestern University, Department of Psychology, July 1977.

\section{REFERENCES}

Brown, J. Recall and recognition. New York: Wiley, 1976.

Underwood, B. J., \& MALMI, R. A. The simultaneous acquisition of multiple memories. In G. H. Bower (Ed.), The psychology of learning and motivation (Vol. 12). New York: Academic Press, 1978.

(Received for publication October 17, 1980.) 